\title{
The Optimal Combination Forecasting Based on ARIMA, VAR and SSM
}

\author{
Beibei Chen ${ }^{1, a}$, Mingyan Jiang ${ }^{1, b^{*}}$ \\ ${ }^{1}$ School of Information Science and Engineering, Shandong University, Jinan, China, 250110 \\ asducbb@163.com, "b Corresponding author: jiangmingyan@sdu.edu.cn
}

Keywords: the optimal combination forecasting model; Artificial Bee Colony algorithm; the Manufacturers' Shipments; ARIMA; VAR; SSM

\begin{abstract}
In order to overcome the defects of a single time series forecasting model and to improve the prediction accuracy, this paper proposes an improved optimal combination model with Artificial Bee Colony algorithm used to solve the optimal weight coefficient automatically. Taking the Manufacturers' Shipments as an example to analyze, we use ARIMA、VAR and SSM to forecast the shipments respectively. Based on these three models, we construct the optimal combination forecasting model. By inspection, it is superior to the other three models in accuracy.
\end{abstract}

\section{Introduction}

One of the purpose of time series analysis is to forecast, which means predicting the future values of a series based on the history of that series and, possibly, other related series of factors [1] .It shows that no single method can be applied to the prediction of all time series. In order to overcome the limitation of the single forecasting model, people combine various forecasting models and use the comprehensive information given by them in proper manner to get the final forecasting result.

The manufacturing industry reflects a country's productivity level, playing a significant role in the economic development of a country. More accurate forecasting of the Manufacturers' Shipments is very important. In this paper, three time series models of ARIMA、VAR and SSM were used to forecast respectively first. Then based on these three models, we automatically get the optimal weights via Artificial Bee Colony algorithm so as to construct the optimal combination model to forecast.

\section{Review of related methodologies}

\subsection{ARIMA model}

The single integrated autoregressive moving average (ARIMA) model which is also known as Box-Jenkins model because of its simplicity, feasibility and flexibility.

If the transferred series is stationary, we can use ARIMA model to forecast. For modeling of seasonal time series beside non-seasonal series, $\operatorname{ARIMA}(p, d, q)(P, D, Q) s$ known as multiplicative ARIMA model is defined as follows:

$$
\begin{aligned}
& \left(1-\phi_{1} B-\phi_{2} B^{2}-\cdots-\phi_{p} B^{p}\right)\left(1-\Phi_{1} B^{s}-\Phi_{2} B^{2 s}-\cdots-\Phi_{P} B^{P s}\right)(1-B)^{d}\left(1-B^{s}\right)^{D} X_{t} \\
= & \left(1-\theta_{1} B-\theta_{2} B^{2}-\cdots-\theta_{q} B^{q}\right)\left(1-\Theta_{1} B^{s}-\Theta_{2} B^{2 s}-\cdots-\Theta_{Q} B^{Q s}\right) \omega_{t}
\end{aligned}
$$


where $\omega_{t}$ is the random variable, $s$ is the periodic term, $B$ is the difference operator as $B\left(X_{t}\right)=X_{t-1},\left(1-B^{s}\right)^{D}$ is the $D$ th seasonal difference measure $s,(1-B)^{d}$ is the $d$ th non-seasonal difference, $p$ and $q$ is the order of non-seasonal autoregressive model and moving average model respectively, $P$ and $Q$ is the order of seasonal autoregressive model and moving average model respectively, $\phi$ and $\theta$ is the parameter of non-seasonal autoregressive model and moving average model respectively, $\Phi$ and $\Theta$ is the parameter of seasonal autoregressive model and moving average model respectively [2].

\subsection{VAR model}

The vector autoregressive (VAR) model is not based on economic theory, whose basic idea is that in each equations of the model, the endogenous variables regress their lagged values so as to estimate the long-term dynamic relationship between variables [3] as follows:

$$
X_{t}=A_{1} X_{t-1}+\cdots+A_{p} X_{t-p}+\Gamma \mu_{t}+\omega_{t},
$$

where $X_{t}$ and $\mu_{t}$ is the endogenous and exogenous variable vector respectively, $\omega_{t}$ is the random variable, $p$ is the lagged rank, $A_{1}, \cdots, A_{p}$ and $\Gamma$ are the coefficient matrix to be estimated.

\subsection{SSM}

State space model (SSM) is often used to estimate the time variable that can not be observed in econometrics. It establishes the relationship between the observable variables and the internal system, and can achieve the purpose of analysis and prediction by estimating the different state vectors.

The general linear normal state space model is defined as follows:

$$
\begin{aligned}
& \alpha_{t+1}=F \alpha_{t}+K \zeta_{t}, \\
& Y_{t}=H \alpha_{t}+\varepsilon_{t},
\end{aligned}
$$

where $\alpha_{t}$ is the variable that can not be observed, $Y_{t}$ is the observed value, $\zeta_{t}$ and $\varepsilon_{t}$ are the random variables, and the three system matrix of $F, K$ and $H$ determine the structure of the model. Eq. (3) is the state equation, describing the state space evolution of a stochastic dynamical system. Eq. (4) is the observation equation, showing that the m-dimensional measurement $Y_{t}$ is subject to a linear transformation of the hidden state $\alpha_{t}$ and is further corrupted by a measurement noise process $\varepsilon_{t}$.

\section{Combination Forecasting Model}

The core issue of combining forecasting is how to distribute the weight of each single prediction model, in order to improve the prediction accuracy effectively. Common methods are arithmetical average method, variance reciprocal method [4], mean square reciprocal method [5], standard deviation method, etc.

\subsection{The Optimal Combination Forecasting model}

This paper uses the optimal weighted method to determine the weight, whose basic idea is to construct the objective function of prediction error according to certain rules, and to determine the optimal weight in the combination forecasting model by solving the optimal value of the objective 
function under certain constraints.

In a certain prediction problem, we set $x$ is the observation object and there are $k$ kinds of methods for forecasting it: $\left\{x_{1}, x_{2}, \cdots, x_{k}\right\}$. The combination forecasting model can be represented as follows:

$$
x(t)=\sum_{i=1}^{k} \omega_{i}(t) x_{i}(t) \quad(t=1,2, \cdots, n)
$$

where $x(t)$ and $x_{i}(t)$ is the predicted value of the $i$ single model and the combination forecasting model in moment of $t$ respectively, $\omega_{i}(t)$ is the weight of the $i$ single in moment of $t$ and meets

$$
\sum_{i=1}^{k} \omega_{i}(t)=1, \omega_{i}(t) \geq 0 \quad(t=1,2, \cdots, n)
$$

On the principle of minimizing the absolute value of the combined prediction error of the sample points, the mathematical expression of optimal combination forecasting model is as follows:

$$
\left\{\begin{array}{c}
\min J_{t}=\left|e_{t}\right|=|x(t)-\hat{x}(t)|=\left|\sum_{i=1}^{k} \omega_{i}(t) e_{i}(t)\right| \\
\text { s.t. } \sum_{i=1}^{k} \omega_{i}(t)=1, \omega_{i}(t) \geq 0
\end{array}\right.
$$

where $e_{i}(t)$ and $e(t)$ is the predicted error of the $i$ single forecasting model and the combination forecasting model in moment of $t$ respectively, $\hat{x}(t)$ is the actual value in moment of $t$.

\subsection{Solving optimal weights by $\mathrm{ABC}$ algorithm}

The solution to the model above is a constrained optimization problem, the Artificial Bee Colony (ABC) algorithm is used to solve the optimal problem on accounts of its high parallelism, randomness, self-adaptability and ease of implementation. The main steps of ABC algorithm are given below [6]:

Send the scouts onto the initial food sources

REPEAT

Send the employed bees onto the food sources and determine their nectar amounts

Calculate the probability value of the sources with which they are preferred by the onlooker bees Send the onlooker bees onto the food sources and determine their nectar amounts

Stop the exploitation process of the sources exhausted by the bees

Send the scouts into the search area for discovering new food sources,randomly

Memorize the best food source found so far

UNTIL(requirements are met)

\section{Simulation}

In order to verify prediction performance of the combination forecasting model, we commit simulation with the model. The raw data, which comes from the U.S. Department of Commerce Web site, are used in the simulation. The data include the Manufacturers' Shipments, New Orders and Total Inventory from February 1992 to June 2015. The data set is divided into training set and test set, in which the training set data is from February 1992 to March 2015, while the test set data is from April 2015 to June 2015. By predicting the test set and calculating the prediction error of each single 
model, we use the ABC algorithm to solve the optimal weight according to Eq. (7) so as to establish the combination forecasting model on the principle of minimizing the absolute value of the combined prediction error of the sample points. The optimal combination weight obtained by using $\mathrm{ABC}$ algorithm is $(0.18240 .56400 .2536)$. To test prediction effect, we introduce the following error indicators.

The mean absolute percent error (MAPE),

$$
M A P E=\frac{1}{n} \sum_{i=1}^{n} \frac{\left|e_{t}\right|}{\hat{x}(t)}=\frac{1}{n} \sum_{i=1}^{n} \frac{|x(t)-\hat{x}(t)|}{\hat{x}(t)}
$$

and the root mean square error (RMSE),

$$
R M S E=\sqrt{\frac{1}{n} \sum_{i=1}^{n}[x(t)-\hat{x}(t)]^{2}}
$$

where $x(t)$ and $\hat{x}(t)$ is predicted value and actual value in moment of $t$ respectively, $n$ is sample number. The comparison of forecasting results by different models is showed in the Tab.1.

From the Tab. 1, we can see that SSM achieved the highest precision among the three single model, but the optimal combination forecasting model can further raise the accuracy of simulation, i.e. the RMSE has declined by 449.3, and the MAPE has declined by 0.153 percent, which means the prediction accuracy is improved by 90 percent. So the proposed model has successfully realized the mutual supplement with each other in terms of advantages of ARIMA、VAR and SSM.

Table 1 Comparison of forecasting results by the four models

\begin{tabular}{|c|c|c|c|c|}
\hline error analysis & ARIMA & VAR & SSM & $\begin{array}{c}\text { the optimal } \\
\text { combination } \\
\text { forecasting model }\end{array}$ \\
\hline MAPE & $0.37 \%$ & $0.21 \%$ & $0.17 \%$ & $0.017 \%$ \\
\hline RMSE & 2158.1 & 1235.8 & 541.2 & 91.9 \\
\hline
\end{tabular}

\section{Conclusions}

This paper presents an improved optimal combination forecasting model based on ARIMA, VAR and SSM. When determining the weights of the model, the Artificial Bee Colony algorithm is used to solve the optimal weight coefficient automatically. Taking the Manufacturers' Shipments as an example to carry on the empirical analysis, we use ARIMA、VAR and SSM to forecast the shipments respectively. Based on these three models, we construct the optimal combination model to forecast. By inspection, the combination model established in this paper is superior to the other three single models. It can get better results in MAPE and RMSE, improving the prediction accuracy and stability of the model. So the proposed model is effective and has certain practical value.

\section{Acknowledgements}

This work was supported by the Shandong Natural Science Foundation (ZR2014FM039).

\section{References}

[1] Claire, Hongyu Pan, et al. Analysis and Application of Time Series: R language [M]. Beijing: Mechanical Industry Press, 2011. 
[2] Karamouz M., Araghinejad sh. Advance Hydrology [M]. Amirkabir University of Technology Press, 2012.

[3] Shiyu Li, Fei Zhang, Zhenglin Wang. Data Analysis: real R language [M]. Beijing: Electronic Industry Press, 2014.

[4] Chengfang Fan, Jianmin Shi. Analysis of Grain Production Cost Forecast based on Holt-Winters and Trend ARMA Combined Model-Taking Corn and Wheat of Shandong Province for Example [J]. Chinese Journal of Agricultural Resources and Regional Planning, vol.3, no.3, pp.45-51, 2014.

[5] Li Zheng, Fengbin Lu, Dongmei Duan, etc. Integration forecast of Chinese pork consumption demand-Empirical based on ARIMA、VAR and VEC models [J]. Systems Engineering - Theory \& Practice, vol.4, no.4, pp.918-925, 2013.

[6] Mingyan Jiang, Dongfeng Yuan. Artificial Bee Colony algorithm and its applications [M]. Beijing: Science press, 2014. 